\title{
BMJ Open Efficacy of Xuebijing Injection for Sepsis (EXIT-SEP): protocol for a randomised controlled trial
}

\author{
Songqiao Liu, ${ }^{\oplus 1}$ Chen Yao, ${ }^{2}$ Junhua Zhang, ${ }^{3}$ Yi Yang, ${ }^{1}$ Haibo Qiu, ${ }^{1}$ On behalf of the \\ EXIT-SEP Investigators
}

To cite: Liu S, Yao C, Zhang J, et al. Efficacy of Xuebijing Injection for Sepsis (EXIT-SEP): protocol for a randomised controlled trial. BMJ Open 2019;9:e028664. doi:10.1136/ bmjopen-2018-028664

- Prepublication history and additional material for this paper are available online. To view these files, please visit the journal online (http://dx.doi. org/10.1136/bmjopen-2018028664).

Received 28 December 2018 Revised 17 July 2019 Accepted 25 July 2019

Check for updates

(c) Author(s) (or their employer(s)) 2019. Re-use permitted under CC BY-NC. No commercial re-use. See rights and permissions. Published by BMJ.

${ }^{1}$ Department of Critical Care Medicine, School of Medicine, Zhongda Hospital, Southeast University, Nanjing, China ${ }^{2}$ Medical Statistics Office, Peking University First Hospital, Beijing, China

${ }^{3}$ Evidence-Based Medicine, Tianjin University of Traditional Chinese Medicine, Tianjin, China

Correspondence to

Dr Haibo Qiu;

haiboq2000@163.com

\section{ABSTRACT}

Introduction Sepsis is a major challenge with high incidence and is associated with high mortality worldwide. Current management of sepsis remains mainly supportive except for treatment with antibiotics. Both basic research and clinical investigation have shown that the Chinese herbal-derived therapeutic Xuebijing (XBJ) injection is beneficial for patients with sepsis. However, the quality of evidence supporting the therapeutic use of XBJ in sepsis is limited. The aim of this trial is to evaluate the Efficacy of Xuebijing Injection for Sepsis, compared with a placebo, on the outcome of patients with sepsis in the intensive care unit (ICU).

Methods and analysis In this multicentre, blinded randomised controlled trial, we are recruiting a total of 1800 subjects who met Sepsis 3.0 criteria. Subjects will be randomised (1:1) to receive XBJ, every 12 hours for 5 days or a matching placebo and usual care. The primary outcome is 28 days all-cause mortality. Secondary outcomes will be the improvement of Sequential Organ Failure Assessment scores, the improvement of the Acute Physiology and Chronic Health Evaluation II score, duration of mechanical ventilation, mortality in ICU and duration of stay in the ICU. Investigators, participants and statisticians will be blinded to the allocated treatment.

Ethics and dissemination This trial has been approved by all ethics committees of the centres that will participate in this trial. The findings of the study will be disseminated in peer-reviewed journals and present at conferences. Once this study is complete, the results of this trial may help provide evidence-based recommendations for complementary therapeutic options for patients with sepsis.

Trial registration number NCT03238742 and ChiCTRIPR-17012713.

\section{INTRODUCTION}

Sepsis is a common and devastating condition that is responsible for more than 6 million deaths worldwide annually and is the leading cause of death in patients who are critically ill. ${ }^{1}$ Half of all patients with sepsis are treated in the intensive care unit (ICU), representing more than $10 \%$ of all ICU admissions. ${ }^{12}$ The WHO reports that sepsis affects more than 30 million people worldwide every year. ${ }^{3}$ The

\section{Strengths and limitations of this study}

- The Efficacy of Xuebijing Injection for Sepsis (EXITSEP) trial is a blinded, randomised controlled study comparing the effect of Xuebijing (XBJ) injection with placebo on outcome in patients with sepsis.

- This is a multicentre study including a wide representation of the Chinese population in the entire country.

- The study will have a sample size large enough to provide high-quality evidence to evaluate the potential benefit of XBJ injection in sepsis.

- One limitation of the study is blinding. Except for drug administrators and a minority of the nurses, the investigators, patients and statisticians were all blinded.

- All participating centres of the EXIT-SEP trial are in China. The result of this study cannot be directly applied in other different population.

burden of sepsis is most likely highest in low/ middle-income countries.

The pathogenesis of sepsis includes an overwhelming inflammatory response, endothelial injury, altered coagulation, dysregulated immune response and sepsis-associated immunosuppression, resulting in acute life-threatening organ dysfunction. During the past 30 years, the understanding of the host immune response has advanced considerably, and in the more than 100 therapeutic clinical trials that have been conducted, no approved treatment options are currently recommended for sepsis. ${ }^{45}$ Although there are improvements in supportive care strategies, mortality still remains unacceptably high. Some adjuvant treatments for sepsis have been developed at great expense but failed to significantly improve mortality. ${ }^{67}$

There is accumulating evidence to suggest that Xuebijing (XBJ) injection has the potential to improve the outcomes for patients with sepsis who are critically ill. XBJ, a Chinese herb-derived therapeutic, has been approved for sepsis treatment in patients 
who are critically ill (China Food and Drug Administration (China FDA); Beijing, China, No Z20040033). The main components of XBJ include amino acids, phenolic acids, flavonoid glycoside, terpene glycoside and phthalides. XBJ has effects on at least 10 sepsis/inflammation pathways and its 21 major active ingredients regulate 550 targets (HRAS, GSK3B, BTK, AK, and so on). ${ }^{8}$ Basic research of XBJ suggests that XBJ has significant immunomodulatory, anticoagulation and anti-inflammatory activity. Animal studies and ex vivo studies have suggested that the effects of XBJ inhibit the expression of HMGB1, inhibit proinflammatory cytokine secretion and ameliorate sepsis-induced lung injury. ${ }^{9-12}$

Clinical trials have demonstrated that XBJ improved outcomes and a meta-analysis showed that XBJ was associated with high survival rate (RR $0.62,95 \%$ CI 0.51 to $\left.0.76, \mathrm{p}<0.000 \quad 01, \mathrm{I}^{2}=0 \%\right) .{ }^{13}{ }^{14}$ These clinical trials, however, were conducted with small sample sizes, even when pooled, limiting the evidence of potential benefit in patients with sepsis. ${ }^{1315}$ Therefore, the large randomised controlled trial (RCT) is needed to access the benefit of XBJ on patients with sepsis. We hypothesise that XBJ will reduce all-cause mortality than the placebo among patients with early sepsis. Thus, we aim to conduct a largescale, blind RCT to evaluate the effects of XBJ on adult patients with sepsis in the ICU.

\section{METHODS \\ Design}

The Efficacy of Xuebijing Injection for Sepsis (EXIT-SEP) study is a multicentre, blind, randomised and placebo-controlled trial. Eligible participants will be randomly assigned to either the XBJ group or the placebo group in a ratio of 1:1. The participants will receive XBJ (XBJ group) or the solvent (placebo group) within 24 hours of enrolment for 5 days. Figure 1 shows the flow chart of the study. This study protocol follows the Standard Protocol Items: Recommendations for Interventional Trials statement recommendations. ${ }^{16}$ The items from the trial

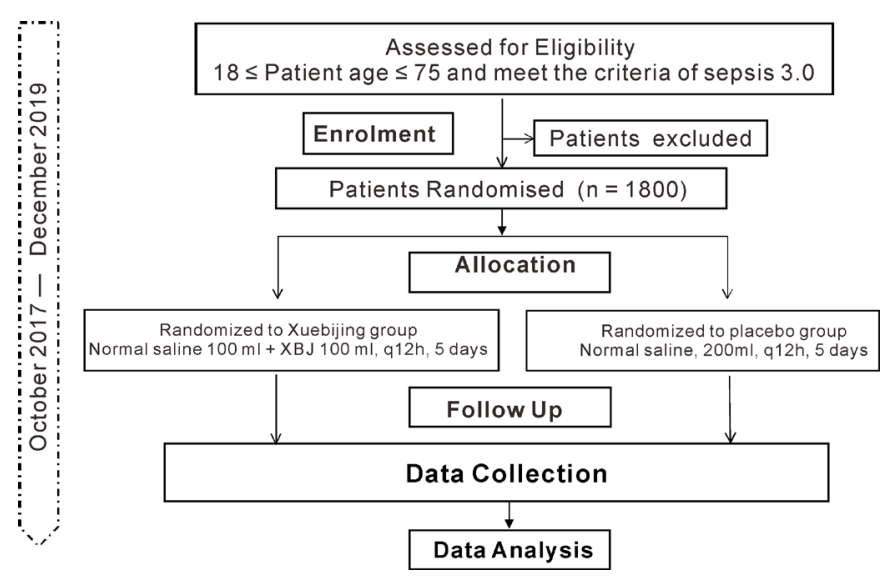

Figure 1 The flow chart of the Efficacy of Xuebijing Injection for Sepsis (EXIT-SEP) study. XBJ, Xuebijing. registration data set are described in the online supplementary material 1 .

\section{Population}

Participant recruitment is currently ongoing at ICUs from the 45 medical centres in China. Patients who fulfil the inclusion criteria and who sign informed consent forms will enter the screening period. Patients with sepsis who meet the exclusion criteria will be excluded before randomisation. The recruitment duration will last for 27 months, from October 2017 to December 2019.

\section{Inclusion criteria}

Patients are eligible for the trial if they are $\geq 18$ and $\leq 75$ years old, meet the criteria of Sepsis 3.0 (The Third International Consensus Definition for Sepsis and Septic Shock) and have Sequential Organ Failure Assessment (SOFA) score of 2-13.

\section{Exclusion criteria}

Patients will be excluded if they fulfil any of the exclusion criteria:

1. Diagnosis of sepsis for more than 48 hours.

2. Pregnant and lactating women.

3. Severe primary disease including unrespectable tumours, blood diseases and HIV.

4. Severe liver and kidney dysfunction (single liver or kidney SOFA score $\geq 3$ points).

5. Use of an immunosuppressant or having an organ transplant within the previous 6 months.

6. Participating in other clinical trials in the previous 30 days.

\section{Ethics and informed consent}

This trial is approved by the Research Ethics Boards at Zhongda Hospital (file number: 2017ZDSYLL025-P01). The research ethics committees of all participant centres approved the study protocol (online supplementary material 2) before randomising patients. The patients' written informed consent (online supplementary material 3) is required at all participating centres. Site investigators will be responsible for obtaining informed consent from study participants or legal representative of the family member. Subject confidentiality will be assured through data anonymisation and controlled access to case report forms (CRF), the electronic data capture system and data sets. Any breaches of confidentiality, study protocol or adverse events (AEs) attributable to this study will be reported to the research ethics committees.

\section{Randomisation and allocation concealment}

Randomisation is generated centrally using an interactive web response system. When an eligible participant has enrolled this subcentre, the drug administrators will log into the central randomisation system, where a random number is produced. The independent drug administrators receive group information based on the random number, and then they assign the study drug to the nurses for administration. 


\section{Interventions}

The participants will receive XBJ (normal saline $100 \mathrm{~mL}+\mathrm{XBJ} 100 \mathrm{~mL}$, every 12 hours, intravenous infusion for $80 \mathrm{~min}$ ) in the XBJ group and the solvent only (normal saline, $200 \mathrm{~mL}$, every 12 hours, also administered for $80 \mathrm{~min}$ ) in the placebo group as previous study. ${ }^{17} \mathrm{XBJ}$ injection includes a $10 \mathrm{~mL} /$ ampule, packaged in five ampules/container. They were manufactured by a Good Manufacturing Practice certified company in China (Tianjin Chase Sun Pharmaceutical, Z20040033). Patients in both groups will receive standard care by the attending physician according to the International Guidelines for Management of Sepsis and Septic Shock. ${ }^{1}$ The patients will receive the study drug within 12 hours of enrolment for a 5-day treatment period. According to the 2016 guidelines on sepsis/sepsis shock, the routine care should include early fluid resuscitation, antimicrobial anticoagulants, nutritional support and other treatment. Ulinastatin and Tanreqing injections are not allowed during the study period. Use of all drugs including antibiotics, if any, should be documented in the CRF.

\section{Blinding}

Photophobic brown colour infusion bags and infusion devices for both groups will be visually inspected by the pharmacy to ensure identical appearance. The independent drug administrators will receive group information based on a random number; they will then assign the study drug to the nurses to administer. The mixture of drugs will be prepared by the nurses in a separate room. This mixture of drugs will be the standard procedure for XBJ, and all the nurses will have experience in performing this task. The nurses will also sign a confidentiality agreement about patient allocation. The participants, as well as all the members of the study and the healthcare team, outcome assessors, will be blinded to the study drug assignment. Data analysis will be performed by a researcher who is blinded to patient allocation.

\section{Outcomes}

The primary outcome will be 28-day all-cause mortality. Secondary outcomes will be the improvement of SOFA scores, the improvement of the Acute Physiology and Chronic Health Evaluation II score (the improvement means the difference of score between the day enrolled in the study and the 6 days), duration of mechanical ventilation, mortality in the ICU and duration of stay in the ICU.

\section{Follow-up}

Data will be recorded during the follow-up period according to the multiple time points. The details are shown in table 1 .

1. Screening period (day 0): before recruitment.

2. Intervention period (days 1-5): data will be recorded every day during follow-up.

3. The period after the intervention (within 28 days after treatment): follow-up at day 28 for survival data. If the patient is discharged, we will contact them via telephone or messaging service.

\section{Sample size calculation}

We determined that enrolment of 1800 participants would provide the trial with $80 \%$ power to detect an absolute difference of six percentage points decrease in 28-day all-cause mortality of $24.3 \%$ with a two-sided $p$ value of 0.05 . This calculation allowed for a rate of withdrawal and loss to follow-up of $15 \%$. We estimated the 28-day mortality rate of $24.3 \%$ in a previous observational Chinese Epidemiological Study of Sepsis (CHESS) study of 2322 patients with sepsis/septic shock with the SOFA score of 2-13 in China.

\section{Study organisation}

The design and implementation of a multicentre study require collaboration among several organisations. Quality control will be undertaken by these organisations: (1) Expert committee: comprises clinical experts, statisticians and quality control experts who will be responsible for clinical research methodology and for resolving the key issues in the implementation of the study. (2) Executive committee: the main staff from the members of the expert committee. The responsibilities of the executive committee include the design of the clinical research trial, selecting the cooperative hospitals and providing a training course including a manual of instructions. (3) Data Safety and Monitoring Board: an independent team that evaluates the safety outcomes and AEs and submits a review proposal. (4) Data management and statistical analysis: performed by trained staff and statisticians. (5) Quality control: sites and researchers will be monitored and inspected regularly by two Clinical Research Organizations (CROs), following the standard protocol throughout the process. This trial will undergo normative monitoring and inspection throughout the entire process.

\section{Data management}

Qualified sites and researchers are crucial factors that ensure the quality of a clinical trial. The subcentres should be qualified by the 'Good Clinical Practice (GCP) training' of the State Food and Drug Administration for compliance in the training. Qualified researchers should understand the detailed contents of the protocol. The data filled in the CRF by researchers should be accurate, complete, timely and reliable. The data will be checked routinely by two CRO(Clinical Research Organization)s.

Data collection will be performed at each participating centre by experienced staff using online electronic data system. ${ }^{18}$ The quality of the data management will be checked by the reliability, access control and traceability of the system. Data management will include baseline characteristics (demographics, comorbidities, inclusion and exclusion criteria, the severity of illness and blood test), the drugs, potential confounder and outcomes. Participants who withdraw from our study for any reason 
Table 1 Schedule of enrolment, interventions, assessments and data collection

\begin{tabular}{|c|c|c|c|c|c|c|c|c|}
\hline \multirow{3}{*}{ Time point } & \multicolumn{8}{|l|}{ Study Period } \\
\hline & \multirow{2}{*}{$\begin{array}{l}\text { Enrollment / Baseline } \\
\text { D0 }\end{array}$} & \multicolumn{5}{|l|}{ Intervention (5 days) } & \multicolumn{2}{|l|}{ Follow-up } \\
\hline & & D1 & D2 & D3 & D4 & D5 & D6 & D28 \\
\hline \multicolumn{9}{|l|}{ Enrollment: } \\
\hline Eligibility screen & $\mathrm{X}$ & & & & & & & \\
\hline Informed consent & $\mathrm{X}$ & & & & & & & \\
\hline Randomization & $\mathrm{X}$ & & & & & & & \\
\hline \multicolumn{9}{|l|}{ Interventions: } \\
\hline Xuebijing injection & & $\mathrm{X}$ & $\mathrm{X}$ & $\mathrm{X}$ & $\mathrm{X}$ & $\mathrm{X}$ & & \\
\hline Normal saline & & $\mathrm{X}$ & $\mathrm{X}$ & $\mathrm{X}$ & $\mathrm{X}$ & $\mathrm{X}$ & & \\
\hline \multicolumn{9}{|l|}{ Assessments: } \\
\hline Demographic data & $\mathrm{X}$ & & & & & & & \\
\hline Etiological examination & $\mathrm{X}$ & & & & & & & \\
\hline Primary disease condition & $\mathrm{X}$ & & & & & & & \\
\hline General condition & $\mathrm{X}$ & & & $\mathrm{x}$ & & & $\mathrm{X}$ & \\
\hline SOFA score & $\mathrm{X}$ & & & $\mathrm{X}$ & & & $\mathrm{X}$ & \\
\hline APACHE II score & $\mathrm{X}$ & & & $\mathrm{x}$ & & & $\mathrm{x}$ & \\
\hline Drugs & $\mathrm{X}$ & $\mathrm{X}$ & $\mathrm{X}$ & $X$ & $\mathrm{X}$ & $\mathrm{X}$ & $\mathrm{X}$ & $x$ \\
\hline Safety & $x$ & & & & & & $\mathrm{X}$ & \\
\hline AEs & & $\mathrm{X}$ & $\mathrm{X}$ & $\mathrm{X}$ & $\mathrm{X}$ & $\mathrm{X}$ & & \\
\hline Mechanical ventilation/CRRT & & & & & & & & $\mathrm{X}$ \\
\hline Duration of stay in the ICU & & & & & & & & $\mathrm{X}$ \\
\hline Time and cost of hospitalisation & & & & & & & & $\mathrm{X}$ \\
\hline Survival condition & & & & & & & & $\mathrm{X}$ \\
\hline
\end{tabular}

AEs, adverse events; APACHE II, Acute Physiology and Chronic Health Evaluation II; CRRT, continuous renal replacement therapy; ICU, intensive care unit; SOFA, Sequential Organ Failure Assessment.

will be followed up and data will be analysed according to the intention-to-treat (ITT) principle. All randomised participants will be followed up until 28 days after randomisation.

\section{Data analysis}

All analyses will be performed according to the ITT principle. Continuous variables will be reported as the means and SDs or as medians and IQRs according to data distribution. Categorical variables will be reported as proportions. We will compare the data on the primary outcome of 28-day all-cause mortality using an unadjusted $\chi^{2}$ test for proportions or a logistic regression model. We will report frequency (percentage) per treatment group with a risk difference and 95\% CI and a corresponding OR and 95\% CI. The missing data of the primary outcome are filled by the worst imputation method, that is, 'death' is used to fill the missing data. The secondary binary and continuous outcomes will be analysed with the use of logistic regression and linear regression, respectively. The rate of death in a time-to-event analysis will be reported with the use of Kaplan-Meier plots, and differences in survival will be tested using a Cox proportional hazards model that includes the group variable. All statistical analyses will be performed using SAS V.9.4 (SAS Institute) with a two-sided $p$ value of less than 0.05 considered significant. No adjustment will be made for multiple comparisons; therefore, secondary outcomes will be interpreted as exploratory.

\section{Patient and public involvement}

No patient or public was involved in the present study. On the completion of this trial, a journal article manuscript will be prepared to present the trial results.

\section{DISCUSSION}

This trial is designed as a multicentre blinded RCT. The completion of this trial will provide evidence on the effectiveness of XBJ injection to reduce mortality of patients with sepsis.

XBJ injection, an intravenous injection approved by the China FDA in 2004, has been evaluated as a promising Chinese herbal-derived therapeutic drug in the management of sepsis, by reducing the inflammatory mediators such as tumour necrosis factor- $\alpha$, interleukin (IL)-1, IL-6 
and IL-8, improving the immune function and protecting the vascular endothelial cells, which correspond to the major pathogenesis of sepsis. ${ }^{19} \mathrm{XBJ}$ injection has pharmacological effects on antagonising endotoxin, inhibiting inflammatory mediators, improving coagulation function and microcirculation, protecting endothelial cells and regulating the immune response. A meta-analysis showed that XBJ reduced all-cause mortality. However, current clinical evidence supporting the efficacy of XBJ in the treatment of sepsis, including RCTs, is subject to limitations such as a non-representative patient population, small sample size and concomitant use of immunomodulatory agents. As a result, the efficacy of XBJ on the mortality of patients with sepsis warrants validation by large, well-conducted RCTs in the ICU.

This trial uses the 28-day mortality as the primary outcome. At the time of planning of this study, hospital mortality rate of patients who met the criteria of Sepsis 3.0 were reported around $20 \%$ in developed countries. Previous studies suggest that ICU patients with sepsis in low/middle-income countries might have a much higher mortality rate than those in high-income countries, possibly due to inadequate resources and quality of care. ${ }^{20}$ Using data from our cross-sectional epidemiology study called 'CHESS', we estimate that the 28-day all-cause mortality will be $31.9 \%$ in the control group and the 28-day mortality rate of $24.3 \%$ in patients of SOFA score no more than 13 . The ICU mortality rate was greater than $60 \%$ in patients with sepsis whose SOFA score was more than 13. Those patients were too severe to detect the benefit of XBJ in ICU. With a sample size of 1800 , this large-scale trial will be adequately powered to test the study hypothesis. The definition of Sepsis 3.0 focuses on organ function, so except for the regular endpoints in clinical trials, we would like to employ more secondary endpoints such as organ function.

There are, however, outcomes that may be influenced by blinding, which can be influenced by discharge decisions. Although the independent drug administrators and a minority of nurses were able to determine the treatment arm, we demonstrated that concealed drug administration achieved satisfactory levels of blinding in a multicentre context. In particular, medications in brown infusion bags and tubes to obscure content were sufficient to achieve blinding.

Recruitment will be completed in December 2019 with a final number of 1800 participants in 45 medical centres in China. The collection of primary endpoint data will conclude in April 2020. With the results of the EXIT-SEP trial, we hope to be able to make evidence-based recommendations for XBJ use for sepsis.

This trial has been approved by Ethics Committeesof all of the centers that participated in this trial. The findings of thestudy will be disseminated at conferences and in peer-reviewed journal.

Contributors $\mathrm{HQ}$ designed the study, drafted the manuscript, edited the manuscript, supervised the study and obtained study funding. SL designed the study, and drafted and edited the manuscript. YY drafted and edited the manuscript. CY designed the study and reviewed the manuscript. JZ edited the manuscript and provided study supervision. The investigators of the EXIT-SEP have participated in the discussion of the protocol and reviewed the manuscript for important intellectual content. All authors have read and approved the final manuscript.

Funding This trial was supported by the Development Center for Medical Science and Technology, National Health and Family Planning Commission of the People's Republic of China (WK-2016-HR-03).

Disclaimer The funder had no role in the design of the protocol, the conduction of the trial, or the analyses or reporting of the data.

Competing interests None declared.

Patient consent for publication Not required.

Ethics approval This study has been approved by the Ethics Committee of Zhongda Hospital, Southeast University (reference number: 2017ZDSYLL025-P01), on 17 May 2017.

Provenance and peer review Not commissioned; externally peer reviewed.

Open access This is an open access article distributed in accordance with the Creative Commons Attribution Non Commercial (CC BY-NC 4.0) license, which permits others to distribute, remix, adapt, build upon this work non-commercially, and license their derivative works on different terms, provided the original work is properly cited, appropriate credit is given, any changes made indicated, and the use is non-commercial. See: http://creativecommons.org/licenses/by-nc/4.0/.

\section{REFERENCES}

1. Rhodes A, Evans LE, Alhazzani W, et al. Surviving sepsis campaign: international guidelines for management of sepsis and septic shock: 2016. Crit Care Med 2017;45:486-552.

2. Singer M, Deutschman CS, Seymour CW, et al. The third International consensus definitions for sepsis and septic shock (Sepsis-3). JAMA 2016;315:801-10.

3. Fleischmann C, Scherag A, Adhikari NKJ, et al. Assessment of global incidence and mortality of Hospital-treated sepsis. current estimates and limitations. Am J Respir Crit Care Med 2016;193:259-72.

4. Delano MJ, Ward PA. Sepsis-Induced immune dysfunction: can immune therapies reduce mortality? J Clin Invest 2016;126:23-31.

5. Gotts JE, Matthay MA. Sepsis: pathophysiology and clinical management. BMJ 2016;353:11585.

6. Paul M, Shani V, Muchtar E, et al. Systematic review and metaanalysis of the efficacy of appropriate empiric antibiotic therapy for sepsis. Antimicrob Agents Chemother 2010;54:4851-63.

7. Annane D. Adjunct therapy for sepsis: how early? Curr Infect Dis Rep 2010;12:361-7.

8. Zhang N, Cheng C, Olaleye OE, et al. Pharmacokinetics-Based identification of potential therapeutic phthalides from XueBiJing, a Chinese herbal injection used in sepsis management. Drug Metab Dispos 2018;46:823-34.

9. Chen S, Dai G, Hu J, et al. Discovery of Xuebijing injection exhibiting protective efficacy on sepsis by inhibiting the expression of HMGB1 in septic rat model designed by cecal ligation and puncture. $A m \mathrm{~J}$ Ther 2016;23:e1819-25.

10. Jiang M, Zhou M, Han Y, et al. Identification of NF-kB inhibitors in Xuebijing injection for sepsis treatment based on bioactivityintegrated UPLC-Q/TOF. J Ethnopharmacol 2013;147:426-33.

11. He X-D, Wang $Y$, Wu $Q$, et al. Xuebijing protects rats from sepsis challenged with Acinetobacter baumannii by promoting annexin A1 expression and inhibiting proinflammatory cytokines secretion. Evid Based Complement Alternat Med 2013;2013:804940.

12. Wang $Q$, Wu $X$, Tong $X$, et al. Xuebijing ameliorates sepsis-induced lung injury by downregulating HMGB1 and RAGE expressions in mice. Evid Based Complement Alternat Med 2015;2015:860259:1-9.

13. Li C, Wang P, Zhang L, et al. Efficacy and safety of Xuebijing injection (a Chinese patent) for sepsis: a meta-analysis of randomized controlled trials. J Ethnopharmacol 2018;224:512-21.

14. Shi H, Hong Y, Qian J, et al. Xuebijing in the treatment of patients with sepsis. Am J Emerg Med 2017;35:285-91.

15. Yin Q, Li C. Treatment effects of xuebijing injection in severe septic patients with disseminated intravascular coagulation. Evid Based Complement Alternat Med 2014;2014:1-6.

16. Chan A-W, Tetzlaff JM, Gøtzsche PC, et al. Spirit 2013 explanation and elaboration: guidance for protocols of clinical trials. BMJ 2013;346:e7586.

17. LIU S-qiao, ZHENG R-qiang, LI M-qin, et al. [Effect of Xuebijing injection treatment on acute respiratory distress syndrome: a 
multicenter prospective randomized control clinical trial]. Zhonghua Yi Xue Za Zhi 2012;92:1017-22.

18. Jiang W, Yu X, Sun T, et al. Adjunctive Ulinastatin in sepsis treatment in China (adjust study): study protocol for a randomized controlled trial. Trials 2018;19:133.

19. Zhang S-wen, Sun C-dong, Wen Y, et al. [Effect of treatment with Xuebijing injection on serum inflammatory mediators and Th1/2 of spleen in rats with sepsis]. Zhongguo Wei Zhong Bing Ji Jiu Yi Xue 2006;18:673-6.

20. Raith EP, Udy AA, Bailey M, et al. Australian, New Zealand intensive care Society centre for $\mathrm{O}$, resource $\mathrm{E}$ : prognostic accuracy of the SOFA score, SIRS criteria, and qSOFA score for in-hospital mortality among adults with suspected infection admitted to the intensive care unit. JAMA 2017;317:290-300. 